\title{
New cosmological solutions and stability analysis in full extended thermodynamics
}

\author{
Luis P. Chimento, Alejandro S. Jakubi \\ Departamento de Física, Facultad de Ciencias Exactas y Naturales , \\ Universidad de Buenos Aires \\ Ciudad Universitaria, Pabellón I, 1428 Buenos Aires, Argentina . \\ Vicenç Méndez \\ Departament de Física. Facultat de Ciències, edifici Cc \\ Universitat Autònoma de Barcelona \\ E-08193 Bellaterra (Barcelona) Spain
}

October 28, 2018

\begin{abstract}
The Einstein's field equations of FRW universes filled with a dissipative fluid described by full theory of causal transport equations are analyzed. New exact solutions are found using a non-local transformations on the nonlinear differential equation for the Hubble factor. The stability of the de Sitter and asymptotically friedmannian solutions are analyzed using Lyapunov function method.
\end{abstract}




\section{Introduction}

Quantum effects played a important role in the early Universe. For instance, vacuum polarisation terms and particle production arise from a quantum description of matter. Some other processes capable of producing important dissipative stresses include interactions between matter and radiation [1] and different components of dark matter [2]. Phenomenologically, these processes may be modeled in terms of a classical bulk viscosity [3].

A relativistic second-order theory of non-equilibrium thermodynamics was developed in [4]. This formulation generalizes the expression of the entropy flux by the inclusion of quadratic terms in dissipative non-equilibrium magnitudes. Another formulation of this theory, called Extended Irreversible Thermodynamics has been made in [5] [6]. There, non-equilibrium magnitudes are introduced and a generalized Gibbs equation including the dissipative magnitudes is employed.

Recently, qualitative analysis of homogeneous cosmological models using the full causal transport theory derived from the extended irreversible thermodynamic [7], [8] has been made to investigate the stability of the particular solutions of the Einstein's field equations. Several authors studied the stability of non flat causal cosmological models using non usual equations of state $p / \theta^{2}=p_{0} x^{l}$, $\zeta / \theta=\zeta_{0} x^{m}[7]$. They are able to get an autonomous system of differential equations in a phase space $(x, y)$ where $x=3 \rho / \theta^{2}$ and $y=9 \Pi / \theta^{2}$. Other authors [8] have studied flat models with the widely used equations of state $p=(\gamma-1) \rho$ and $\zeta=\alpha \rho^{m}$. They have got an autonomous system in the phase space $(H, \dot{H})$. In both studies, always the linearization technique near stationary solutions in the phase space have been used. Some other papers where the full version of the transport equation has been used are [9] [10] [11] [12].

In this work, together with another one [13], new exact cosmological solutions of full causal models are found and the asymptotic stability of solutions present in non-causal and truncated causal models is investigated using the direct method of Lyapunov. Few authors have used this method to analyze the stability of causal models though it provides useful information of the dynamical behaviour of the system, not only near the stationary solutions but also far away from them [13] [15] [16]. In the present paper we assume that the cosmic fluid is described locally by the equation of state $p=n T$, while in the paper [13] a power-law relationship between the equilibrium temperature and energy density was assumed.

This work summarizes as follows. In section II we state the basic equations of the model, they are solved in section III for $m=1 / 2$, the two-parameter families of solutions are classified and their asymptotic behaviors are analyzed. Also, the thermodynamics of these solutions is considered. In section IV we study the stability conditions for de Sitter and asymptotically Friedman solutions for $m \neq 1 / 2$. Finally in section $\mathrm{V}$ we summarize the main conclusions of the paper. 


\section{Hydrodynamic description for the cosmic fluid. Basic equations}

For the fluid approach to remain valid, the mean interaction time $t_{c}$ of the fluid particles must be much less than the expansion rate. This requires, $t_{c} \ll H^{-1}$, where $H$ is the Hubble expansion rate. If the fluid is in or close to equilibrium, then this restriction ensures that the fluid has a well-defined local temperature. For a cosmic fluid without shear viscosity or heat flow, the energy momentum tensor is

$$
T_{a b}=(\rho+p+\Pi) u_{a} u_{b}+(p+\Pi) g_{a b},
$$

where $u_{a}$ is the four velocity, $\rho$ the energy density, $p$ the equilibrium pressure and $\Pi$ the bulk viscous pressure. In an expanding fluid, the dissipation due to $\Pi$ leads to a decrease in kinetic energy and therefore in pressure, so $\Pi \leq 0$. The particle flow vector $N^{a}$ is given by $N^{a}=n u^{a}$ where $n$ is the particle number density. In absence of vector and tensor dissipation, the entropy four-flux will be of the form $S^{a}=S_{\text {eff }} n^{a}$ where $S_{\text {eff }}$ is the effective non-equilibrium specific entropy which must be positive.

In the Israel/Stewart theory

$$
S_{e f f}=S-\frac{\tau}{2 n T \zeta} \Pi^{2}
$$

where $\tau$ is the relaxation time, $T$ is the local-equilibrium temperature and $\zeta$ is the coefficient of bulk viscosity. The conservation laws $N_{; a}^{a}=0$ and $T_{; b}^{a b}=0$ imply

$$
\dot{n}+3 H n=0 \text { and } \dot{\rho}+3 H(\rho+p+\Pi)=0
$$

respectively, where $3 H=u_{; a}^{a}$ is the fluid expansion and $\dot{n}=n_{, a} u^{a}$. Combining equations (3) with the Gibbs equation for the local-equilibrium variables $S$ and $T$

$$
T d S=d\left(\frac{\rho}{n}\right)+p d\left(\frac{1}{n}\right)
$$

we get

$$
\dot{S}=-\frac{3 H \Pi}{n T} .
$$

From (2) and (3) we find

$$
S_{; a}^{a}=-\frac{\Pi}{T}\left[3 H+\frac{\tau}{\zeta} \dot{\Pi}+\frac{1}{2} \Pi T\left(\frac{\tau u^{a}}{\zeta T}\right)_{; a}\right]
$$


for the entropy production rate. The simplest way to guarantee the second law of thermodynamics $S_{; a}^{a} \geq 0$ is establishing the transport equation

$$
\Pi+\tau \dot{\Pi}=-3 \zeta H-\frac{1}{2} \tau \Pi\left(3 H+\frac{\dot{\tau}}{\tau}-\frac{\dot{T}}{T}-\frac{\dot{\zeta}}{\zeta}\right) .
$$

for $\Pi$, leading to $S_{; a}^{a}=\Pi^{2} / \zeta T$.

We need to specify equations of state $\rho=\rho(T, n)$ and $p=p(T, n)$ for the cosmological fluid. In order to have a simple model, we assume that the cosmic viscous fluid may be locally described by an equilibrium equation of state that has the approximated form of an ideal gas and the $\gamma$-law:

$$
\begin{aligned}
p & =n T \\
\rho & =\frac{1}{\gamma-1} n T .
\end{aligned}
$$

Note that both $T$ and $n$ are independent variables and only in the equilibrium limit the particle number density depends exclusively on $T$.

From (3) and (8) the expression for the temperature is

$$
T=\frac{\gamma-1}{n_{0}} R^{3} \rho
$$

We specialise to a flat FRW universe, where the field equations are

$$
\begin{aligned}
H^{2} & =\frac{1}{3} \rho, \\
3\left(\dot{H}+H^{2}\right) & =-\frac{1}{2}(\rho+3 p+3 \Pi),
\end{aligned}
$$

where $H \equiv \dot{R} / R$ is the Hubble factor, $R(t)$ the cosmic scale factor of the Robertson-Walker metric, $8 \pi G=c=1$ and an overdot denotes differentiation respect to time $t$. The relaxation time $\tau$ is related to the bulk viscosity by 14

$$
\tau=\frac{\zeta}{v^{2} \gamma \rho}
$$

where $v$ is the dissipative contribution to the speed of sound. By causality $v^{2} \leq$ $2-\gamma$. The bulk viscosity coefficient is often taken to be $\zeta=\alpha \rho^{m}$ where $\alpha$ is a positive constant.

An other interesting restriction is that, since a dissipative expansion is nonthermalising, the rate for some interaction $\left(\sim \tau^{-1}\right)$, which is crucial to maintaining equilibrium, must remain lower that the expansion rate $H$, so that the fluid cannot adjust sufficiently rapidly to the changes in temperature induced by expansion [12]. 
Finally, using (7), (9), (10) and (11) we get, for the evolution of $H$, the equation

$$
H \ddot{H}+\frac{v^{2} \gamma}{\delta} \dot{H} H^{3-2 m}-2 \dot{H}^{2}+\frac{3 v^{2} \gamma H^{4}}{2}\left(\frac{\gamma}{\delta} H^{1-2 m}-3\right)=0
$$

where $\delta=\alpha 3^{m-1}$, and we are assuming that the Universe is expanding, that is $H>0$. Using (5), (8) and (10) we get, after integrating

$$
S(t)=S_{0}+\frac{1}{\gamma-1} \ln \left(H^{2} R^{3 \gamma}\right)
$$

\section{Exact solution for $m=1 / 2$}

The Einstein's field equations of FRW universes filled with a dissipative fluid described by both the truncated and non-truncated causal transport equations can be linearized and solved for a power-law fluid when $m=1 / 2$ [13 [15. In what follows, we shall find the general solution for a fluid that obeys (8). In this case setting $m=1 / 2$ in (12) we find

$$
H \ddot{H}+\frac{v^{2} \gamma}{\delta} \dot{H} H^{2}-2 \dot{H}^{2}+\frac{3 a H^{4}}{2}\left(\frac{\gamma}{\delta}-3\right)=0 .
$$

With the change of variables $H=1 / s, t=\delta \sigma / v^{2} \gamma$, equation (14) becomes into

$$
\frac{d^{2} s}{d \sigma^{2}}+\frac{1}{s} \frac{d s}{d \sigma}+\frac{\mu}{s}=0
$$

where $\mu=\left(3 \delta / 2 v^{2} \gamma\right)(3 \delta-\gamma)$.

Equation (15) is a particular case of the most general equation

$$
\ddot{s}+\alpha_{1} f(s) \dot{s}+\alpha_{2} f(s) \int f(s) d s+\alpha_{3} f(s)=0 .
$$

which linearizes through the nonlocal transformation [15] [17] [18] [19]

$$
z=\int f(s) d s \quad \eta=\int f(s) d t
$$

becoming

$$
z^{\prime \prime}+\alpha_{1} z^{\prime}+\alpha_{2} z+\alpha_{3}=0
$$

where the ' indicates derivative with respect to the new variable $\eta$. In our case $\alpha_{1}=1, \alpha_{2}=0, \alpha_{3}=\mu, f(s)=1 / s$ and the nonlocal transformation is then

$$
z=\ln s \quad \eta=\frac{v^{2} \gamma}{\delta} \int \frac{d t}{s}
$$

Thus, integrating (18) 


$$
z(\eta)=c_{1}-\mu \eta-c_{2} e^{-\eta}
$$

we obtain the general solution of (14) in parametric form

$$
\begin{gathered}
H(\eta)=H_{0} \exp \left(\mu \eta+c_{2} e^{-\eta}\right) . \\
\Delta t=\frac{\delta}{v^{2} \gamma H_{0}} \int d \eta \exp \left(-\mu \eta-c_{2} e^{-\eta}\right) .
\end{gathered}
$$

where $\Delta t=t-t_{0}$, and $H_{0}, c_{2}, t_{0}$ are arbitrary integration constants. The scale factor can also be found in terms of $\eta$ as

$$
R(\eta)=R_{0} \exp \left(\frac{\delta}{v^{2} \gamma} \eta\right)
$$

where $R_{0}$ is another integration constant. In this case $\zeta \sim H$ and $\tau \sim H^{-1}$.

Below, we show explicitly exact solutions and the asymptotic behaviour of the general solution.

\subsection{General solution for $\mu=1$}

In the particular case $\mu=1$, Equation (16) has an additional form invariance symmetry. Then, introducing the transformation [18]

$$
s^{-1}=\frac{\dot{R}}{R}=-\frac{\delta}{v^{2} \gamma} \frac{w^{-1}}{\int w^{-1} d t}
$$

into (15) we find $w^{\prime \prime}(\sigma)=0$ and $w(\sigma)=c_{1} \sigma+c_{2}$. Finally, the two-parameter family of solutions is given by

$$
R(t)=R_{0}(c \pm \ln |\Delta t|)^{-\delta / v^{2} \gamma},
$$

where $\delta=\left(\gamma+\sqrt{\gamma^{2}+8 v^{2} \gamma}\right) / 6$, and $c$ is an arbitrary integration constant. There exist solutions that expand so quickly that the scalar curvature diverges when the scale factor diverges and they become infinite in a finite amount of proper time. Some of these singular solutions, those with minus, have a finite time span and particle horizons. Also, we have the time reversal of the above solutions. These with plus lead to a shrinking universe. In both cases, the dynamical information is enough in order to select the optimal physical solutions. Non-thermalising condition requires in this case $v<\frac{1}{\sqrt{3}} \sqrt{1+2 / 3 \gamma}$. 


\subsection{General solution for $\mu=0$}

We note first that in this case $(\gamma / 3=\delta)$ equation (12) admits a de Sitter stable solution $H=H_{0}$, where $H_{0}$ is arbitrary [8]. On the other hand, for $H \neq H_{0}$, equation (15) can be integrated twice by quadratures

$$
\Delta t=-\frac{1}{3 v^{2}} \int \frac{d H}{H^{2}}\left(\ln \frac{H}{H_{0}}\right)^{-1}
$$

and we find these two-parameter families of solutions in implicit form

$$
\begin{gathered}
\Delta t=\frac{1}{3 v^{2} H_{0}} \mathrm{E}_{1}\left(\ln \frac{H}{H_{0}}\right) \\
\Delta t=\frac{1}{3 v^{2} H_{0}} \mathrm{E}_{1}\left[\left(\frac{R}{R_{0}}\right)^{-3 v^{2}}\right]
\end{gathered}
$$

for $H / H_{0}>1$, and

$$
\begin{gathered}
\Delta t=-\frac{1}{3 v^{2} H_{0}} \operatorname{Ei}\left(-\ln \frac{H}{H_{0}}\right) \\
\Delta t=-\frac{1}{3 v^{2} H_{0}} \operatorname{Ei}\left[\left(\frac{R}{R_{0}}\right)^{-3 v^{2}}\right]
\end{gathered}
$$

for $H / H_{0}<1$, and where $\mathrm{E}_{1}$ and $\mathrm{Ei}$ are exponential integrals. Solutions of both families have an asymptotically de Sitter stage for large times, while their respective initial behavior (singular or nonsingular) follows those of the solutions with $\mu=1$. Non-thermalising condition requires here $v<1 / \sqrt{3}$ and the second law of thermodynamics does not impose additional restrictions.

\subsection{Particular solution for $c_{2}=0$}

If $c_{2}=0$ in (20) then $H(\eta=0)=H_{0}$ and $R(\eta=0)=R_{0}$ and we find that $H=2 /(3(\gamma-3 \delta) \Delta t)$ and the scale factor is given by

$$
R(t)=R_{0} \Delta t^{\beta}
$$

where $\beta=2 / 3(\gamma-3 \delta)$. This solution is also found if we set $H=\beta / \Delta t$ in (14). If $\beta>0$, that is, for $\delta<\gamma / 3$, the Universe starts from a Big-Bang singularity and then expands, while for $\delta>\gamma / 3$ the scale factor diverges in a finite amount of proper time. For $\delta=\gamma / 3$, we have $\mu=0$ and $z=c_{1}$. In this case $R=R_{0} e^{H_{0} t}$ is the de Sitter solution. Non-thermalising condition and $S_{\text {eff }} \geq 0$, at late times, require that for $v<1 / \sqrt{3}, \alpha$ must be in the range $\sqrt{3} v^{2} \gamma<\alpha<\gamma / \sqrt{3}$.

For

$$
v<\frac{1}{\sqrt{3}} \sqrt{1-\frac{2}{3 \gamma}} \text { and } \frac{\gamma}{\sqrt{3}}-\frac{2 \sqrt{3}}{9}<\alpha<\frac{\gamma}{\sqrt{3}}
$$


there exist inflationary thermodynamically consistent power-law solutions given by (31). For

$$
\sqrt{3} v^{2} \gamma<\alpha<\frac{\gamma}{\sqrt{3}}-\frac{2 \sqrt{3}}{9}
$$

non-inflationary friedmannian thermodynamically consistent solutions given by (31) exist. We conclude that whenever

$$
v<\frac{1}{\sqrt{3}} \sqrt{1-\frac{2}{3 \gamma}}
$$

inflationary power-law and non-inflationary scenarios are thermodynamically consistent.

For $\beta=1 / 2$, that is $\alpha=\sqrt{3}(3 \gamma-4) / 9$ for $4 / 3<\gamma<2$, the universe behave as if were filled by a perfect fluid and were radiation dominated. For $\beta=2 / 3$, that is, $\alpha=\sqrt{3}(\gamma-1) / 3$, the universe behave as if were filled by a perfect fluid and were matter dominated.

\subsection{Analysis of the solutions}

Here we analyze the behavior of the general solution (21)- (22). We begin by studying its asymptotic behavior near the singularities and for large times.

For $H \rightarrow \infty, s \rightarrow 0$ and $z \rightarrow-\infty$. This occurs in two cases. If $\mu>0, z \simeq-\mu \eta$ for $\eta \rightarrow+\infty$. Then, using (19), we find that $H \sim e^{\mu \eta}=-1 /(\mu \sigma)$. So, there is a two-parameter family of solutions with an explosive singularity at a finite time whose leading behavior is given by (31). Also, $H$ diverges when $\eta \rightarrow-\infty$ and $c_{2}>0$. In this case, $H \sim \exp \left(c_{2} e^{-\eta}\right)$ and there is a two-parameter family of solutions representing a Universe beginning to from a Big-Bang singularity as

$$
R(t) \sim|\ln | \Delta t||^{-3 v^{2}} \text { for } \Delta t \rightarrow 0
$$

The opposite extreme is $H \rightarrow 0$. In this case $s \rightarrow \infty$ and $z \rightarrow+\infty$. If $\mu<0$ we obtain $z \simeq|\mu| \eta$ for $\eta \rightarrow+\infty$. Using the (19) again, we obtain $H=e^{-|\mu| \eta}=1 /(|\mu| \sigma)$. So there exists a biparametric family of asymptotically Friedmann solutions with leading behavior given by (31). Furthermore, these solutions are stable, and (31) is an attractor.

When $\eta \rightarrow-\infty$, two different behaviors occur depending on whether $c_{2}<0$ or $c_{2}=0$. In the first case, $H \sim \exp \left(c_{2} e^{-\eta}\right)$ and there is a two-parameter family of solutions representing a Universe beginning to evolve as

$$
R(t) \sim|\ln | \Delta t||^{-3 v^{2}} \text { for } t \rightarrow-\infty
$$

In the second case the solution is (31).

The two-parameter families of solutions can be classified according to their number of singularities. 
There is a family of singularity-free solutions which begin at $t=-\infty$ and their behavior in the far future is Friedmann or have a final de Sitter stage. These solutions do no have particle horizons.

There is a family of solutions that begin as in the previous case but end at an explosive singularity. These solutions do not have particle horizons. Also there is a family of solutions that begin at a Big-Bang singularity and have a final Friedmann or de Sitter behavior.

Finally, there is a family of solutions with a finite time-span that begin at a Big-Bang singularity and end at an explosive singularity.

\subsection{Thermal consequences}

Inserting the general solution (21), (22) in (9)

$$
T=3 \frac{\gamma-1}{n_{0}} R_{0}^{3} H_{0}^{2} \exp \left[\frac{3 \delta}{v^{2} \gamma}(1+3 \delta-\gamma) \eta+2 c_{2} e^{-\eta}\right]
$$

we can investigate the evolution of the temperature for the different families of solutions studying the limits near the singularities and for large times. For $t \rightarrow \infty$, the temperature vanishes provided that $\alpha<\sqrt{3}(\gamma-1) / 3$, so the scale factor grows asymptotically slower than $t^{2 / 3}$. The above condition implies that $\mu<-(\gamma-1) /(2 \gamma v)<0$. This shows that, although a power-law inflationary scenario is possible, the temperature grows unboundly in the far future. For evolutions with a singularity in the past, we have a hot Big-Bang beginning of the Universe because the temperature diverges at the singularity. On the other hand, for evolutions ending at an explosive singularity, the temperature diverges as a negative power of the proper time interval to the singularity. There are two two-parameter families of solutions which have a cool beginning with $R=0$ in the far past. These contain a set of solutions that have a cool ending provided $\alpha<\sqrt{3}(\gamma-1) / 3$, otherwise they have a hot final stage. With a fine-tuning of the parameters which corresponds to solutions with the asymptotical behavior $R \sim t^{2 / 3}$ when $t \rightarrow \infty, T \rightarrow 3 R_{0}^{3} H_{0}^{2}(\gamma-1) / n_{0}$. In this particular case, the solution (31) has a constant temperature.

\section{$4 \quad$ Stability analysis for $m \neq 1 / 2$}

We will make use of the method of the Lyapunov function 16 to investigate the asymptotic stability of the de Sitter and asymptotically Friedmann solutions that occur in the noncausal and truncated causal models [15], as well as in the full causal model a with power-law equation of state [13]. 


\subsection{Stability of the de Sitter solution}

Equation (12) admits a de Sitter solution [8]

$$
H_{0}=\left(\frac{3 \delta}{\gamma}\right)^{1 /(1-2 m)} \quad(m \neq 1 / 2) .
$$

To study its stability we make the change of variable $H=s^{-1}$ in (12) which becomes into

$$
\ddot{s}+\frac{v^{2} \gamma}{\delta} s^{2 m-2} \dot{s}-\frac{3 v^{2} \gamma^{2}}{2 \delta} s^{2 m-2}+\frac{9 v^{2} \gamma}{2 s}=0
$$

multiplying by $\dot{s}$ one may rewrite (36) as

$$
\frac{d}{d t}\left(\frac{1}{2} \dot{s}^{2}+V(s)\right)=D(s, \dot{s})
$$

where the term $\dot{s}^{2} / 2$ represents the kinetic energy term of the system and $V(s)$ the potential energy term. The dissipative term $D(s, \dot{s})$ is negative definite for all $s$. One may calculate these terms and finds

$$
\begin{aligned}
& V(s)=-\frac{3 v^{2} \gamma^{2}}{2 \delta} \frac{s^{2 m-1}}{2 m-1}+\frac{9 v^{2} \gamma}{2} \ln s \\
& D(s, \dot{s})=-\frac{v^{2} \gamma}{\delta} \dot{s}^{2} s^{2 m-2}
\end{aligned}
$$

The potential $V(s)$ has a unique minimum for $s>0$ at $s_{0}=H_{0}^{-1}$ for $m<1 / 2$, while for $m>1 / 2$ it becomes a maximum. Thus we find that an exponential inflationary regime is asymptotically stable for $t \rightarrow \infty$ for any initial condition $H>0$ provided that $m<1 / 2$ (node or stable focus), but this regime becomes unstable for $m>1 / 2$ (saddle point). This result improves over previous studies based in small perturbations about the solution (35) [8].

\subsection{Stability of the asymptotically Friedmann solution}

For $m>1 / 2$ it is easy to check that (12) admits a solution whose leading term is $2 /(3 \gamma t)$ for $t \rightarrow \infty$. This suggests to make the change of variables

$$
H=\frac{1}{u(z) t}, \quad t^{2 m-1}=z
$$

in (12) which takes the form

$$
\frac{d}{d z}\left[\frac{1}{2} u^{\prime 2}+U(u, z)\right]=D\left(u, u^{\prime}, z\right)
$$

where $^{\prime} \equiv d / d z$. We consider that $u$ lies in a neighbourhood of $u_{0} \equiv 3 \gamma / 2$. Thus, when $z \rightarrow \infty$ 


$$
U(u, z)=\frac{v^{2} \gamma}{2 \delta(2 m-1)^{2}}\left(\frac{u^{2 m}}{m}-\frac{3 \gamma u^{2 m-1}}{2 m-1}\right) \frac{1}{z}+O\left(\frac{1}{z^{2}}\right)
$$

for $m \neq 0$,

$$
U(u, z)=\frac{v^{2} \gamma}{\delta}\left(\ln \frac{u}{u_{0}}+\frac{3 \gamma}{2 u}\right) \frac{1}{z}+O\left(\frac{1}{z^{2}}\right)
$$

for $m=0$, and

$$
D\left(u, u^{\prime}, z\right)=-\frac{v^{2} \gamma}{\delta(2 m-1)} u^{2 m} u^{\prime 2}+O\left(\frac{1}{z}\right) .
$$

As $U(u, z)$ has a unique minimum at $u_{0}$ for any $m \neq 1 / 2$, and $D\left(u, u^{\prime}, z\right)$ is negative definite for $m>1 / 2$, we find that solutions with leading Friedmann behaviour $R \sim t^{2 /(3 \gamma)}$ when $t \rightarrow \infty$ are asymptotically stable for $m>1 / 2$, but become unstable for $m<1 / 2$. This behavior has already been found in the truncated theory and the power-law model [20], [13].

\section{Conclusions}

A cosmological model for a causal viscous fluid with an equation of state like that of an ideal gas is analyzed carefully. Our main result is that many qualitative features of this model are similar to those of the full causal model with a powerlaw equation of state for the temperature and the truncated model.

Applying a non-local integral transformation to the nonlinear differential equation for the expansion rate it linearizes, and we find the general solution of this model for $m=1 / 2$. Due to viscous effects, these new exact solutions contain a two-parameter family that avoid the singularity and do not have particle horizons. This interesting evolution has already been found in the full causal theory, with a power-law equation of state for the equilibrium temperature [13.

Among the set of solutions for some values of the parameters we have found explicit exact ones that give the qualitatively behavior of parametric solutions for $\mu>0$. Also, there are solutions that begin at a Big-Bang singularity and may represent a power-law inflationary stage or a matter or radiation dominated era.

For $m=1 / 2$, the splitting of the large time asymptotic behavior of solutions in terms of $\operatorname{sgn}(\gamma-3 \delta)$ follows closely that of the power-law model and is the same as in the truncated model, which in its turn resembles the classification for the noncausal solutions. As in the power-law case, no family of solutions with extrema is found, and this appears to be the main consequence of the full theory. On the other hand, a logarithmic leading behavior appears near the BigBang singularity and in the remote past. This kind of evolution has not been observed previously for these models. We note that the rate of expansion in this logarithmic Big-Bang is larger than in a regular power-law one. 
The asymptotic behavior of solutions for this model was analyzed using the method of Lyapunov for $m \neq 1 / 2$. We have shown that friedmannian solutions are an attractor set of the biparametric family of solutions for $m \geq 1 / 2$, while they are unstable for $m<1 / 2$. This information is not available via the stability analysis by linearization [8]. So we have improved this analysis, obtaining more information away from the stationary solutions. Also we have found that an exponential inflationary stage is asymptotically stable for $m \leq 1 / 2$ while it is unstable for $m>1 / 2$. Here also, the same structural behavior as in the powerlaw, truncated [20] and non-causal models is observed.

It has been conjectured that due to the use of causal truncated or noncausal theories, viscosity-driven exponential inflationary solutions are spurious [9]. There, a cosmological model containing a dissipative Boltzmann gas is studied. We show here that this conclusion does not generalize to models described by another equation of state.

\section{Acknowledgments}

We thank to Diego Pavón for his interesting comments about the results of this paper. One of us ( $\mathrm{LPCh}$ ) wants to acknowledge the hospitality of the Departament de Física, Facultat de Ciències, of the Universitat Autònoma de Barcelona, where part of this work was done. This was partially supported by project 3091/92 CONICET and by the Dirección General de Investigación Científica y Técnica of the Spanish Ministry of Education and Science under grant PB94-0718.

\section{References}

[1] Weinberg S 1971 Astrophys. J. 168175

[2] Pavón D and Zimdahl W 1993 Phys. Lett. A 179261

[3] Hu B. L. 1982 Phys. Lett. A 90375

[4] Israel W and Steward J M 1976 Phys. Lett. 58A 213

[5] Pavón D, Jou D and Casas-Vázquez J 1982 Ann. Inst. H. Poincare A 3679

[6] Jou D, Casas-Vázquez J and Lebon G 1993 Extended Irreversible Thermodynamics (Springer Verlag, Berlin) 
[7] Coley A.A., van der Hoogen R J , Maartens R. 1996 Phys. Rev. D 541393

[8] Méndez V. and Triginer J. 1996 J. Math. Phys. 37, 2906 (1996)

[9] Hiscock W A and Salmonson J 1991 Phys. Rev. D 433249

[10] Zakari M and Jou D 1993 Phys. Rev. D 481597

[11] Romano V and Pavón D 1994 Phys. Rev. D 502575

[12] Maartens R 1995 Class. Quantum Grav. 121455

[13] Chimento L P and Jakubi A S 1996 Class. Quantum Grav. (in press)

[14] R. Maartens, in Proceedings of Hanno Rund Workshop, to appear; astro$\mathrm{ph} / 9609119$

[15] Chimento L P and Jakubi A S 1993 Class. Quantum Grav.10 2047

[16] Krasovskii N N 1963 Stability of Motion (Stanford University Press: Stanford)

[17] Chimento L P and Jakubi A S 1996 Phys. Lett. A 212320

[18] Chimento L P 1996 J. Math. Phys. (in press)

[19] Aguirregabiria J M and Chimento L P 1996 Class. Quantum Grav. 133197

[20] Pavón D, Bafaluy J and Jou D 1991 Class. Quantum Grav. 8347 